\title{
Somatostatin Receptor-Targeted Radiopeptide Therapy in Treatment-Refractory Meningioma: Individual Patient Data Meta-analysis
}

\author{
Christian Mirian ${ }^{1,2}$, Anne Katrine Duun-Henriksen ${ }^{3}$, Andrea Maier ${ }^{1}$, Maria Møller Pedersen ${ }^{1}$, Lasse Rehné Jensen ${ }^{1}$, \\ Asma Bashir ${ }^{4}$, Thomas Graillon ${ }^{5}$, Maya Hrachova ${ }^{6}$, Daniela Bota ${ }^{6,7}$, Martjin van Essen ${ }^{8}$, Petar Spanjol ${ }^{2}$, Christian Kreis ${ }^{2}$, \\ Ian Law ${ }^{4}$, Helle Broholm ${ }^{9}$, Lars Poulsgaard ${ }^{1}$, Kåre Fugleholm ${ }^{1,10}$, Morten Ziebell ${ }^{1}$, Tina Munch ${ }^{1,10,11}$, Martin A. Walter*2, \\ and Tiit Mathiesen*1,10,12
}

\begin{abstract}
${ }^{1}$ Department of Neurosurgery, Rigshospitalet, Copenhagen University Hospital, Copenhagen, Denmark; ${ }^{2}$ Department of Nuclear Medicine, University Hospital of Geneva, Geneva, Switzerland; ${ }^{3}$ Statistics and Pharmacoepidemiology, Danish Cancer Society Research Center, Copenhagen, Denmark; ${ }^{4}$ Department of Clinical Physiology, Nuclear Medicine and PET, Copenhagen University Hospital, Copenhagen, Denmark; ${ }^{5}$ APHM, Department of Neurosurgery, La Timone Hospital, Marseille, France; ${ }^{6}$ Department of Neurology, UC Irvine Medical Center, Irvine, California; ${ }^{7}$ Department of Neurosurgery, UC Irvine Medical Center, Irvine, California; ${ }^{8}$ Department of Clinical Physiology, Sahlgrenska University Hospital, Gothenburg, Sweden; ${ }^{9}$ Department of Neuropathology, Copenhagen University Hospital, Copenhagen, Denmark; ${ }^{10}$ Department of Clinical Medicine, University of Copenhagen, Copenhagen, Denmark; ${ }^{11}$ Department of Epidemiology Research, Statens Serum Institut, Copenhagen, Denmark; and ${ }^{12}$ Department of Clinical Neuroscience, Karolinska Institute, Stockholm, Sweden
\end{abstract}

Somatostatin receptor (SSTR)-targeted peptide receptor radionuclide therapy (PRRT) represents a promising approach for treatmentrefractory meningiomas. Methods: We performed an individual patient data meta-analysis, including all published data on meningioma patients treated with SSTR-targeted PRRT. The main outcomes were toxicity, response to treatment, progression-free survival (PFS), and overall survival (OS). We applied the Kaplan-Meier method to estimate survival probabilities and report incidence rates per 100 person-years. We applied Cox proportional hazards models to determine the effect of covariates. Results: We screened 537 papers and identified 6 eligible cohort studies. We included a total of 111 patients who had treatment-refractory meningioma and received SSTR-targeted PRRT. Disease control was achieved in $63 \%$ of patients. The $6-\mathrm{mo}$ PFS rates were $94 \%, 48 \%$, and $0 \%$ for World Health Organization grades I, II, and III, respectively. The risk of disease progression decreased by $13 \%$ per $1,000-\mathrm{MBq}$ increase in the total applied activity. The 1 -y OS rates were $88 \%, 71 \%$, and $52 \%$ for World Health Organization grades I, II, and III, respectively. The risk of death decreased by $17 \%$ per $1,000-\mathrm{MBq}$ increase in the total applied activity. The main side effects comprised transient hematotoxicity, such as anemia in $22 \%$ of patients, leukopenia in $13 \%$, lymphocytopenia in $24 \%$, and thrombocytopenia in $17 \%$. Conclusion: To our knowledge, this individual patient data meta-analysis represents the most comprehensive analysis of the benefits of and adverse events associated with SSTRtargeted PRRT for treatment-refractory meningioma. The treatment was well tolerated, achieved disease control in most cases, and showed promising results regarding PFS and OS.

Received May 14, 2020; revision accepted Jul. 29, 2020.

For correspondence or reprints contact: Christian Mirian, Department of Neurosurgery, Rigshospitalet, Copenhagen University Hospital, Blegdamsvej

9, DK-2100 Copenhagen, Denmark.

E-mail: Christian.mirian.larsen@regionh.dk

${ }^{*}$ Contributed equally to this work.

Published online Aug. 28, 2020.

COPYRIGHT (C) 2021 by the Society of Nuclear Medicine and Molecular Imaging.
Key Words: treatment-refractory meningioma; progressive meningioma; peptide receptor radionuclide therapy; somatostatin receptor

J Nucl Med 2021; 62:507-513

DOI: 10.2967/jnumed.120.249607

$\mathbf{M}$ glial primary neoplasm (I). Low-grade meningiomas (World Health Organization [WHO] grade I [WHO-I]) are usually benign and typically display indolent behavior (2), whereas high-grade meningiomas (WHO-II and WHO-III) have higher rates of recurrence $(1,3)$. Meningiomas of all grades may show multiple recurrences and become refractory to treatment (4).

Therapeutic options for recurrent and progressive meningiomas are limited to high-dose radiation and repeated surgery, often with unsatisfactory results. Several approaches with targeted therapy and cytotoxic chemotherapy have been investigated in recent decades but have failed to demonstrate significant efficacy (2). Thus, new treatment modalities are urgently needed.

Most meningiomas express a high density of somatostatin receptor (SSTR) subtypes, making them susceptible to SSTR-targeted peptide receptor radionuclide therapy (PRRT), such as DOTA-Tyr ${ }^{3}$ octreotide (DOTATOC) and DOTA-Tyr ${ }^{3}$-octreotate (DOTATATE) labeled with the $\beta^{-}$-emitting radioisotopes ${ }^{90} \mathrm{Y}$ and ${ }^{177} \mathrm{Lu}(5,6)$.

The aims of the present work were to systematically evaluate evidence for SSTR-targeted PRRT by analyzing toxicity, response to treatment, progression-free survival (PFS), and overall survival (OS) via an individual patient data (IPD) meta-analysis of all published data on patients subjected to SSTR-targeted PRRT.

\section{MATERIALS AND METHODS}

The study adhered to the Preferred Reporting Items for Systematic Review and Meta-Analyses of individual participant data (PRISMA-IPD) Statement (7). 


\section{Literature Search}

We searched PubMed, Embase, the Cochrane Library, and ClinicalTrials. gov on June 17, 2019, using 2 different search strings: first, the MeSH term "octreotide" or "somatostatin" in combination with "meningioma," and second, "meningioma" in combination with either "radiopeptide," "radionuclide," "octreotide," or "somatostatin." Two authors independently screened abstracts and full texts, settling all disagreements by consensus.

\section{Study Selection}

We included studies investigating patients treated with any radiolabeled somatostatin analog for otherwise treatment-refractory or inoperable meningiomas. We excluded case reports and abstracts but did not impose restrictions on language. Treatment-refractory meningioma was defined as recurrent or progressive meningioma for which control failed despite multiple attempts with conventional treatment modalities, including surgery, fractioned or stereotactic radiotherapy, or chemotherapy. Hence, therapeutic options were considered to be exhausted by the treating physicians before the initiation of SSTRtargeted PRRT. The fraction of patients with progressing tumors at the time of PRRT initiation is unknown; however, eligibility criteria in 1 study comprised tumor progression within 12 mo before PRRT ( $n=$ 34) (8). Tumors were considered inoperable because of anatomic location, comorbidity, or a patient's refusal.

\section{Outcomes and Data Extraction}

We contacted the authors of each study $(n=6)$ and obtained the following IPD: age, WHO tumor grade, total activity applied (in megabecquerels), number of treatment cycles, best obtained radiologic treatment response, PFS, and OS.

As the data were either completely accessible online or received in a completely anonymized form (i.e., the data could not be tracked to any patient), we were not required by Danish law to obtain institutional review board approval.

\section{Quality of Evidence and Risk of Bias}

We rated the quality of evidence according to the Grading of Recommendations Assessment, Development and Evaluation and applied ROBINS-I, a tool developed by the Cochrane Collaboration for assessing risk of bias in nonrandomized studies of interventions (Supplemental Tables 1 and 2; supplemental materials are available at http://jnm. snmjournals.org) (9).

\section{Data Synthesis and Statistical Analysis}

We pooled data into 1 cohort for simultaneous analysis, thus applying the 1-stage approach in accordance with the PRISMA-IPD Statement (7). We extracted adverse events as reported in the original studies. All studies addressed hematotoxicity but applied different assessment schemes: Common Terminology Criteria for Adverse Events (CTCAE) version 3.0, CTCAE version 4.0, and WHO criteria for hematotoxicity from 1979 (10-12). CTCAE versions 3.0 and 4.0 were identical in terms of hematotoxicity and thus were comparable. Two studies that applied WHO criteria for hematotoxicity reported grade 1 hematotoxicity exclusively. Since WHO criteria for hematotoxicity and CTCAE version 4.0 use identical laboratory data points (levels of hemoglobin, granulocytes, leukocytes, and thrombocytes), we allocated all patients with WHO grade 1 hematotoxicity to CTCAE version 4.0 grade 1 .

We generated a weighted estimate of the radiologic treatment response. Despite different radiologic assessment schemes, each radiologic evaluation included stable disease, progression of disease, and partial response. We applied a random-effects model and quantified heterogeneity as low, moderate, and high, corresponding to $\mathrm{I}^{2}$ values of $25 \%, 50 \%$, and $75 \%$, respectively (13).

We estimated the probabilities of PFS and OS at $6,12,18$, and 24 mo using the Kaplan-Meier method. The end of follow-up was the date of death, loss to follow-up, or individual study termination. We estimated progression and mortality rates per 100 person-years for each WHO grade and subsequently compared all incidence rates as ratios. We used univariate and multivariate Cox proportional hazard regression models to estimate the association between risk of progression or death and the covariates age (at diagnosis), total applied activity, and WHO grade (I, II, III, and unknown). The univariate estimates were adjusted only for the effect of each individual study center (center effect), whereas the multivariate estimates were adjusted for all covariates, including the center effect.

We tested for nonlinear effects of the continuous covariates age and total applied activity with restricted cubic spline regression and found that a linear relationship was adequate in both cases $\left(\chi^{2} ; P>0.05\right)$. We evaluated the assumption of proportionality for all models with visual inspection of Schoenfeld residuals, concluding that all covariate effects were proportional.

The results of a subgroup $(n=82)$ analysis of the effects of ${ }^{90} \mathrm{Y}$ DOTATOC $(n=47 ; 57 \%)$ versus ${ }^{177}$ Lu-DOTATATE plus ${ }^{90}$ Y-DOTATOC $(n=35 ; 43 \%)$ on OS are provided in Supplemental Table 3.

Finally, we applied a likelihood ratio test $\left(\chi^{2}\right)$ to evaluate for potential effect modification.

\section{RESULTS}

\section{Study Selection}

The search yielded 537 publications (Fig. 1). We identified and reviewed 9 studies for eligibility. Two studies were considered duplicates because they were based on the same patients and used data from included studies $(5,14)$. Two studies combined SSRTtargeted PRRT with fractionated external-beam radiotherapy and were excluded from the analysis $(15,16)$. Thus, we identified 6 eligible studies $(5,6,8,17-19)$.

\section{Study Characteristics}

We contacted all corresponding authors. All specific IPD of interest were accessible from the publication of 3 studies $(5,6,17)$, and 3 authors provided original raw data from their respective studies $(8,18,19)$.

Two studies were phase 2 clinical trials $(6,8)$. One study examined the effect of SSTR-targeted PRRT prospectively over a 6-y period (18), whereas 2 studies evaluated the effect retrospectively over a 6-y period (5) and a 2-y period (17). Finally, 1 study monitored patients prospectively with routine scans every sixth month (19). The SSTR-targeted PRRT was ${ }^{90}$ Y-DOTATOC, ${ }^{177} \mathrm{Lu}$-DOTATOC, ${ }^{177}$ Lu-DOTATATE, or combinations thereof $(5,6,8,17-19)$. Patient inclusion criteria were similar across the studies and comprised a confirmed histologic diagnosis, tumor uptake on SSTR scintigraphy or PET/CT, disease progression or recurrence despite treatment, and a lack of further therapeutic options.

We included 111 patients who received SSTR-targeted PRRT between 1998 and 2015. Thirty-seven patients (33\%) had WHO-I, 29 patients (26\%) had WHO-II, and 19 patients (17\%) had WHO-III. For the remaining 26 patients (23\%), the grade could not be assessed (i.e., unknown). Nineteen of the 111 patients (17\%) had inoperable tumors (5 from Gerster-Gilliéron et al. (6), 9 from Marincek et al. (8), 3 from Bartolomei et al. (18), and 2 from van Essen et al. (19)).

The median total applied activity for the entire cohort was $12,950 \mathrm{MBq}$ (range, 1,688-29,772). Figure 2 depicts the range and median total applied activity per WHO grade. The total applied activity was independent of WHO grades $\left(\chi^{2} ; P=0.16\right)$.

Data on PFS could not be retrieved for 35 patients $(8,19)$ Therefore, the PFS analysis was based on 76 patients. Data on OS could 


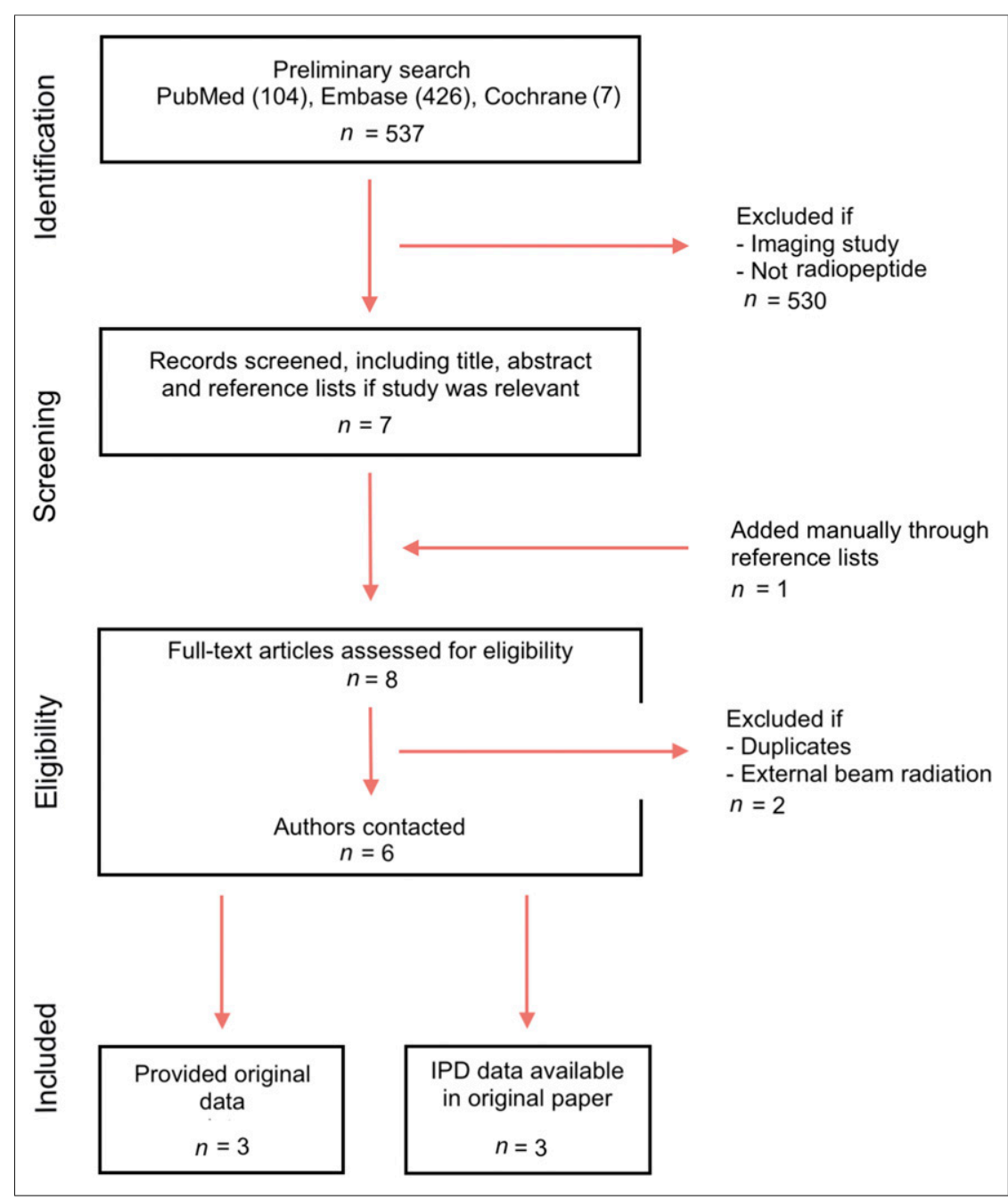

FIGURE 1. Flow diagram of study selection.

not be retrieved for 1 patient (19). Thus, the OS analysis comprised 110 patients. Study and patient characteristics are listed in Supplemental Table 4.

\section{Toxicity}

The most frequently observed adverse event was grade 1 or 2 transient hematotoxicity (anemia [22\% of patients], leukopenia

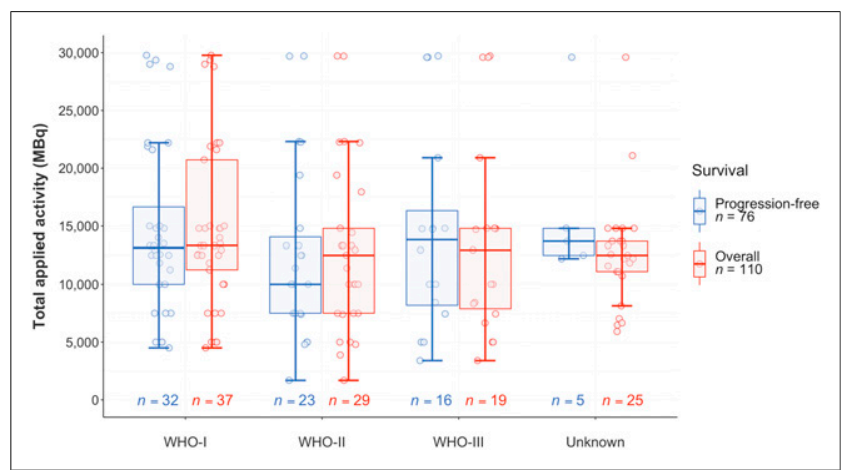

FIGURE 2. Box plots of individual total applied activity per WHO grade of patients included in PFS and OS analysis.
[13\%], lymphocytopenia [24\%], and thrombocytopenia [17\%]) (Supplemental Table 5).

Other transient adverse events, based on CTCAE version 4.0, comprised 1 case of grade 4 renal toxicity (8), 1 case of seizure, 1 case of cerebral edema, and 1 case of grade 2 renal toxicity that occurred 14 mo after the treatment (6). Permanent adverse events comprised 1 case each of grade 1 renal toxicity (18), alopecia (5), and pituitary insufficiency (5).

\section{Treatment Response}

Three distinct radiologic assessment protocols were applied to assess the radiologic treatment response (Southwest Oncology Group [SWOG] (17-19), RECIST version $1.1(6,8)$, and Macdonald (5)). Sixty-four patients $(58 \%)$ achieved stable disease, 45 patients (41\%) experienced progression, and 2 patients (2\%) had partial remission (Fig. 3A). A random-effects model estimated that $63 \%$ of patients (95\% CI, 0.45-0.81) experienced disease control. However, the model showed considerable and significant heterogeneity $\left(\mathrm{I}^{2}=77.3 \% ; P<0.001\right.$ ) (Fig. 3B). Subsequently, we stratified the data for the different radiologic assessment protocols, revealing moderate $\left(\mathrm{I}^{2}=60.9 \%\right)$ and nonsignificant $(P=0.11)$ heterogeneity for RECIST version $1.1(6,8)$ and high $\left(\mathrm{I}^{2}=\right.$ $83.0 \%)$ and significant $(P<0.001)$ heterogeneity for SWOG (17-19).

\section{PFS}

In total, 34 of 76 patients $(45 \%)$ experienced progression during 117 personyears of follow-up. The cohort received a median of 3 (range, 1-6) treatment cycles. The PFS rates are listed in Supplemental Table 6. The 6-mo PFS (PFS6) and 12-mo PFS rates were $61 \%$ (95\% CI, 50-72) and 53\% (95\% CI, 42-65) for all grades combined, respectively (Fig. 4A). We subsequently stratified the data on the basis of WHO grade and found PFS6 rates of 94\% (95\% CI, 85-100), 48\% (95\% CI, 27-68), and $0 \%$ for WHO-I, WHO-II, and WHO-III meningiomas, respectively (Figs. 4B-4D).

We estimated progression rates per 100 person-years (Fig. 5A) and observed gradually increasing rates corresponding to higher WHO grades (Fig. 5C). In the multivariate analysis, the rate of progression was significantly associated with the total applied activity, with a hazard ratio (HR) of 0.87 (95\% CI, 0.79-0.95) per $1,000-\mathrm{MBq}$ increase; these data indicated that the rate of progression decreased by $13 \%$ per $1,000-\mathrm{MBq}$ increase (Table 1 ). Figure $6 \mathrm{~A}$ predicts the adjusted correlation between PFS and total applied activity. The reference was set to the median total applied activity of 12,540 MBq (Supplemental Table 4); each HR must be interpreted relative to this reference.

There was no significant interaction between total applied activity and WHO grade, indicating that the effect of SSTR-targeted PRRT on PFS was not modified by WHO grade $\left(\chi^{2} ; P=0.7\right)$. 


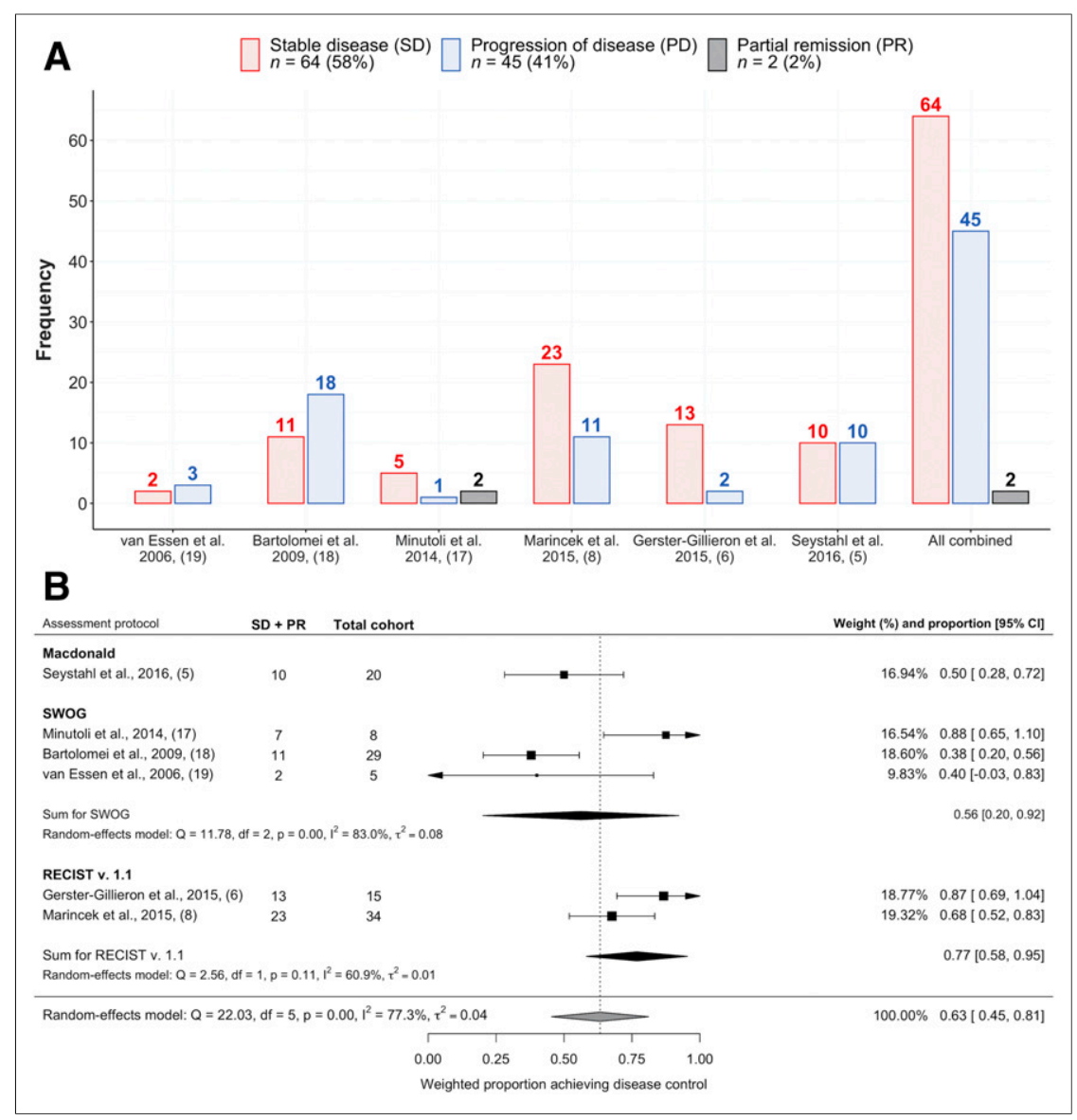

FIGURE 3. (A) Cross-sectional observations of best radiologic treatment response obtained by each individual study and all studies combined. (B) Forest plot of random-effects model estimating weighted proportion of patients achieving SD or better. Overall and subgroup estimates were based on radiologic assessment scheme applied. v. = version.

\section{OS}

Forty-five of 110 patients (41\%) died during 263 person-years of follow-up. The cohort received a median of 2 (range, 1-6) treatment cycles. Survival times are summarized in Supplemental Table 6. The 6-mo OS and 12-mo OS (OS12) rates for all tumors were $89 \%$ (95\% CI, 83-95) and 78\% (95\% CI, 70-86), respectively (Fig. 4A). Stratifying the data by WHO grade, we found OS12 rates of $88 \%$ (95\% CI, 77-99), 71\% (95\% CI, 53-88), and $52 \%$ (95\% CI, 28-77) for WHO-I, WHO-II, and WHO-III, respectively (Figs. 4B and 4C).

The mortality rate for cases with unknown tumor grades was 11.4 per 100 person-years, which was slightly higher than the 8.1 deaths per 100 person-years observed for WHO-I tumors but markedly lower than the 31.1 and 43.1 deaths per 100 personyears observed for WHO-II and WHO-III tumors, respectively (Fig. 5B).

The mortality rate ratio for WHO-I versus unknown was 0.73 (95\% CI, 0.29-1.84), and the mortality rate ratio for WHO-II versus WHO-III was 0.72 (95\% CI, 0.34-1.64); these data indicated no significant difference between these groups (Fig. 5C). The mortality rate increased with increasing grades. Thus, the lowest mortality rate ratio was 0.19 (95\% CI, 0.08-0.46), for WHO-I versus WHO-III (Fig. 5C). In the multivariate analysis, the risk of death was significantly associated with the total applied activity, with an HR of 0.83 (95\% CI, 0.76-0.90) per $1,000-\mathrm{MBq}$ increase; these data indicated that the risk of death decreased by $17 \%$ per $1,000-\mathrm{MBq}$ increase (Table 1). Figure 6B predicts the adjusted correlation between OS and total applied activity. The reference was set to the median total applied activity of 12,950 MBq (Supplemental Table 4); each HR must be interpreted relative to this reference.

There were no interactions between total applied activity and WHO grade, suggesting that the effect of SSTR-targeted PRRT on OS was not modified by WHO grade $\left(\chi^{2} ; P=0.09\right)$.

\section{DISCUSSION}

The present IPD meta-analysis represents a comprehensive analysis of the benefits of and adverse events associated with SSTRtargeted PRRT for treatment-refractory meningioma. The results can be summarized as follows. First, SSTR-targeted PRRT was well tolerated in patients with treatmentrefractory meningioma. All included studies concluded good overall tolerability of PRRT. Most patients experienced mild transient hematotoxicity, which was manageable in all cases. Second, SSTR-targeted PRRT resulted in disease control in most patients with treatment-refractory meningioma. Nevertheless, the respective random-effects model was associated with considerable and significant heterogeneity. Third, SSTRtargeted PRRT resulted in favorable PFS (for low-grade tumors primarily) and OS in patients with treatment-refractory meningioma. Specifically, PFS6 rates were 94\%, 48\%, and 0\% for patients with WHO-I, WHO-II, and WHO-III meningiomas, whereas the corresponding OS12 rates were $88 \%, 71 \%$, and $52 \%$, respectively. Finally, we established a prediction model for total

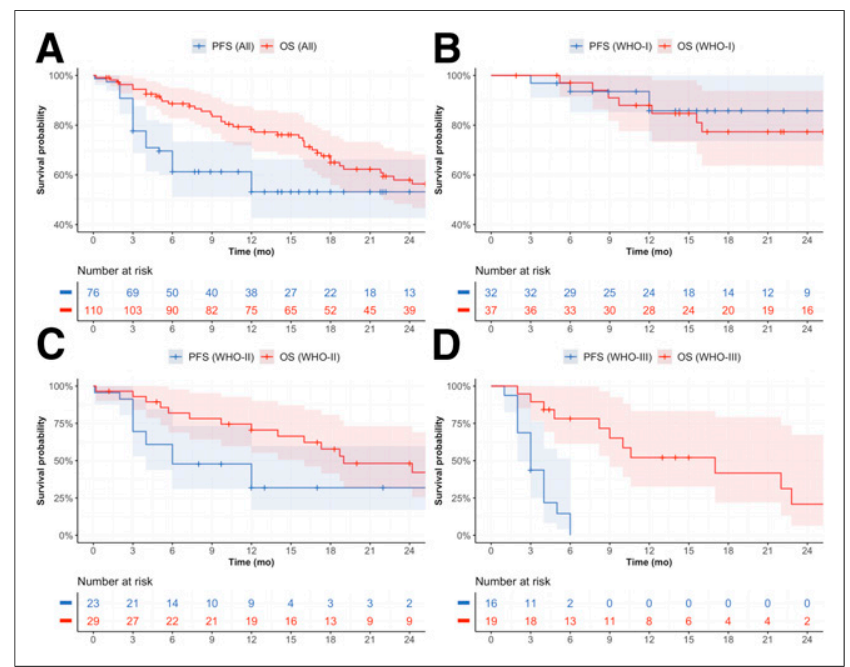

FIGURE 4. Kaplan-Meier curves. (A) PFS and OS of all cases. (B) PFS and OS for WHO-I meningioma. (C) PFS and OS for WHO-II meningioma. (D) PFS and OS for WHO-III meningioma. 


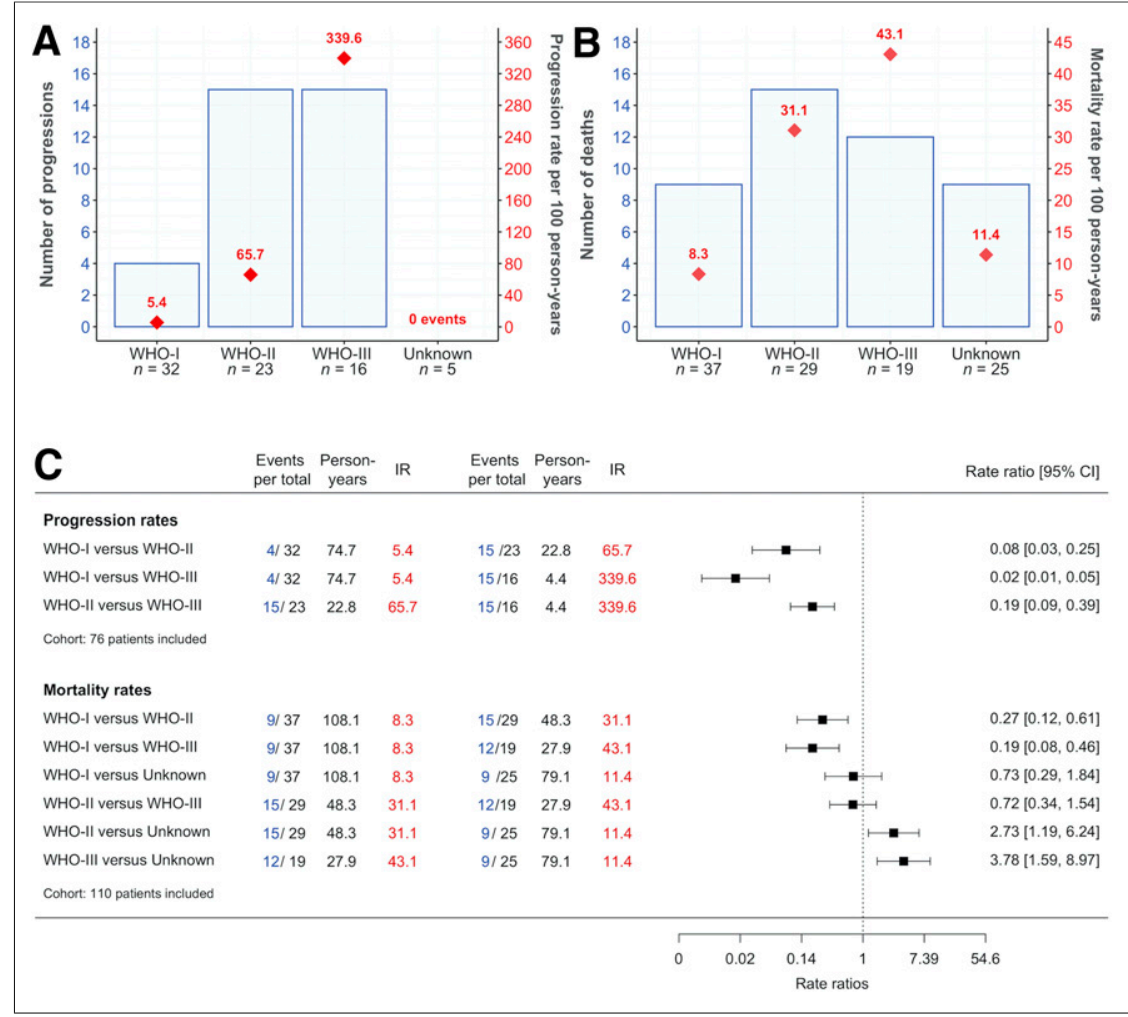

FIGURE 5. (A) Progression per WHO grade. Number of progressions (left-hand $y$-axis) and progression rate per 100 person-years (right-hand $y$-axis). (B) Mortality per WHO grade. Number of deaths (left-hand $y$-axis) and mortality rate per 100 person-years (right-hand $y$-axis). (C) Incidence rate ratios (IRR) of progression and mortality.

applied activity and the correlation with progression or death, both indicating clinical benefits.

\section{Strength and Limitations}

The primary strength of the present study is the $100 \%$ rate of inclusion of original data from previously published cases of SSTRtargeted PRRT for treatment-refractory meningioma. We pooled the studies through the one-stage method for IPD meta-analysis, thus making it possible to adjust and explore the data in a manner different from that used in a meta-analysis of aggregated data.
The present study also has limitations. First, none of the included studies was randomized. However, our primary objective was not to compare different treatments but to analyze toxicity, response to treatment, PFS, and OS after PRRT; these are typical objectives of phase 1 or 2 trials $(21)$. The relevance of this approach is supported by a meta-analysis of 61 cancer drugs, in which phase 3 or 4 studies did not significantly increase the detection of toxicities if the original phase 1 trial included more than 60 patients (22). Thus, the inclusion of 111 patients in the present study should have been adequate for detecting relevant toxicities.

Second, the studies applied 3 different radiologic assessment protocols. Three studies used SWOG, 2 used RECIST version 1.1, and 1 used Macdonald. The radiologic protocols are not completely comparable. One study found a $21 \%$ discordance when SWOG and RECIST version 1.1 criteria were applied to the same 80 patients (23). Different radiologic assessment protocols along with the evaluation of tumors at nonequivalent time points might partially explain the heterogeneity observed in the weighted estimate of $63 \%$ disease control. Consensus on assessment methods would improve external validity in future studies. There is already consensus in neurooncology and neuroimaging societies that the Response Assessment in Neuro-Oncology (RANO) criteria should serve as the standard response criteria $(24,25)$.

Third, the included studies used different $\beta^{-}$-emitting radionuclides and somatostatin analogs. We did not aim for a comparative analysis, since comparative efficacy can be established only in larger trials (20). The feasibility of pooling different PRRTs is supported by experience with neuroendocrine tumors. Both ${ }^{90}$ Y-DOTATOC and ${ }^{177} \mathrm{Lu}$-DOTATOC improved survival in patients with neuroendocrine tumors, with no significant difference in the median OS (25).

TABLE 1

PFS and OS According to Total Applied Activity, Age at Diagnosis, and WHO Grade

\begin{tabular}{|c|c|c|c|c|}
\hline \multirow[b]{2}{*}{ Covariate } & \multicolumn{2}{|c|}{ PFS $(n=76)$} & \multicolumn{2}{|c|}{ OS $(n=110)$} \\
\hline & Univariate & Multivariate* & Univariate & Multivariate* $^{*}$ \\
\hline Total applied activity $/ 1,000-\mathrm{MBq}$ increase & $0.84(0.77-0.91)$ & $0.87(0.79-0.95)$ & $0.83(0.77-0.90)$ & $0.83(0.76-0.90)$ \\
\hline Age at diagnosis/10-y increase & $1.11(0.86-1.44)$ & $1.08(0.79-1.47)$ & $1.29(1.00-1.66)$ & $1.47(1.07-2.00)$ \\
\hline WHO grade I & Reference & Reference & Reference & Reference \\
\hline WHO grade II & $9.06(2.85-28.80)$ & $8.09(1.50-26.11)$ & $2.45(1.05-5.72)$ & $2.32(0.95-5.63)$ \\
\hline WHO grade III & $31.17(8.50-114.34)$ & $25.78(6.76-98.40)$ & $4.61(1.78-11.95)$ & $2.28(0.78-6.72)$ \\
\hline Unknown grade & $\mathrm{NA}^{\dagger}$ & $\mathrm{NA}^{\dagger}$ & $2.58(0.86-7.71)$ & $2.45(0.73-8.24)$ \\
\hline \multicolumn{5}{|c|}{$\begin{array}{l}\text { *Adjusted for age at diagnosis, total applied activity, WHO grade, and center effect. } \\
\text { †Unknown grade }(n=5) \text { was omitted from model; NA = not analyzed. } \\
\text { Data are HRs, with } 95 \% \text { Cls in parentheses. }\end{array}$} \\
\hline
\end{tabular}




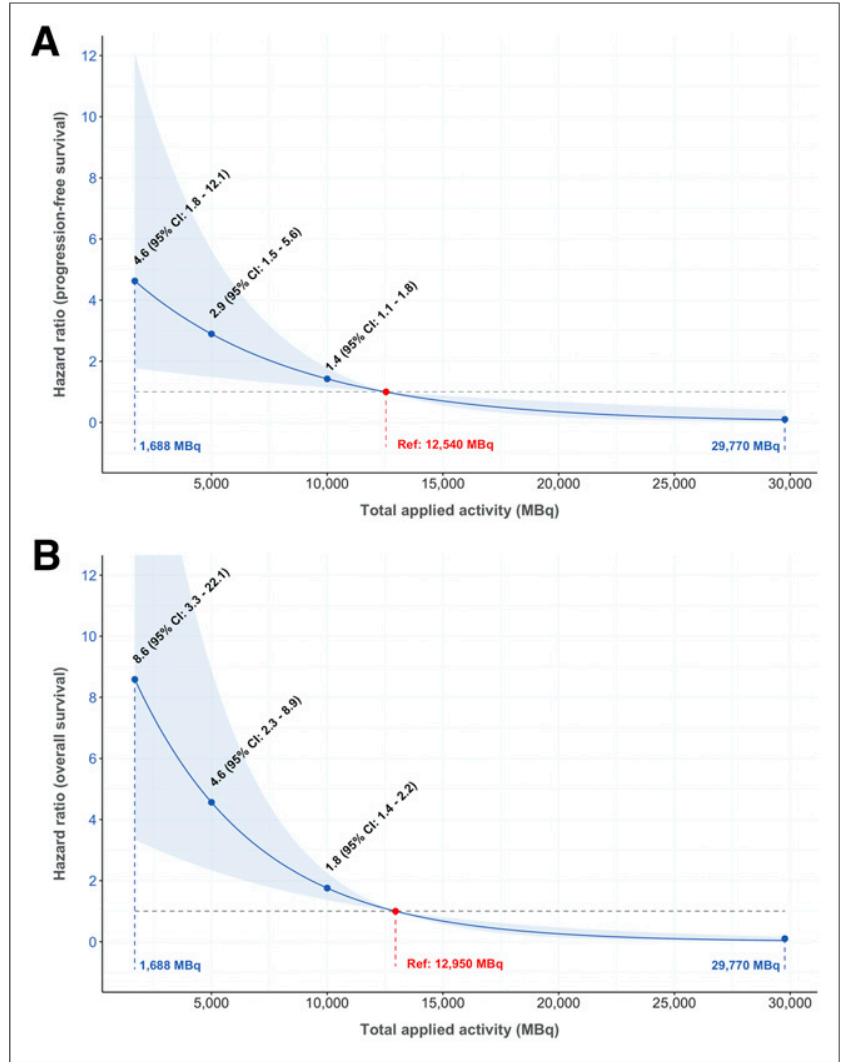

FIGURE 6. (A) Correlation between total applied activity and risk of progression. Model was adjusted to median total applied activity $(12,540 \mathrm{MBq})$, age at diagnosis, WHO grade (reference [Ref] set to WHO-I), and center effect. Unknown grade $(n=5)$ was omitted from model. (B) Correlation between total applied activity and risk of death. Model was adjusted to median total applied activity $(12,950$ $\mathrm{MBq}$ ), age at diagnosis, WHO grade (reference set to WHO-I), and center effect.

Finally, some prognostic covariates were not accessible $(4,26)$. The extent of surgery is often prognostically important but was not included in the IPD (27). Given our highly selected cohort of patients who had treatment-refractory meningiomas with uniformly progressive behavior, we would not assume this parameter to markedly affect the results. Patients with treatment-refractory meningiomas have dismal prognoses, and it is unlikely that comorbidities such as cardiovascular disease, diabetes, or other cancers would have significantly affected the estimated OS.

\section{Comparison with Results from Literature}

Our results on toxicity are in agreement with accumulating evidence from studies that validated SSTR-targeted PRRT as a well-tolerated therapy for neuroendocrine tumors, with only transient and manageable adverse events $(20,28-30)$.

The pooled findings of an antitumoral response are in agreement with those of an excluded study (15) that combined PRRT with external-beam radiotherapy, with seemingly even better effects. Kreissl et al. included 10 treatment-refractory meningiomas (7 WHO-I, 2 WHO-II, and 1 unknown) (15). Six patients received ${ }^{90}$ Y-DOTATOC, 4 received ${ }^{177} \mathrm{Lu}$-DOTATATE, and all patients received external-beam radiotherapy ranging between 40 and $60 \mathrm{~Gy}$. All patients had stable disease, including 1 partial remission and 1 complete remission (15). These results suggested better disease control than with PRRT alone and indicated the potential of combining PRRT with external-beam radiotherapy.

The present results for PFS are promising in comparison with those obtained with other therapies. A RANO review of 47 studies of surgery- and radiation-refractory meningiomas reported a weighted PFS6 after treatment(s) with a variety of different agents (2). WHO-I meningiomas had a PFS6 rate of $29 \%(95 \% \mathrm{CI}$, 20.3-37.7) for all treatments combined, whereas the weighted PFS6 rate for WHO-II and WHO-III meningiomas combined was $26 \%$ (95\% CI, 19.3-32.7). The RANO review proposed that therapies achieving PFS6 rates of at least $50 \%$ for WHO-I meningiomas and at least 35\% for WHO-II and WHO-III meningiomas combined would be of potential clinical interest (2). The present study found PFS6 rates of 94\%, 48\%, and 0\% for WHO-I, WHO-II, and WHO-III meningiomas, respectively, and $37.7 \%$ for WHO-II and WHO-III meningiomas combined; these data compared favorably with the RANO-proposed criteria for treatment-refractory WHO-I, WHO-II, and WHO-III meningiomas.

Furthermore, the present results for OS are also promising. The RANO review observed that OS was less commonly reported and varied greatly among the included studies (2). For treatmentrefractory WHO-I meningiomas, the median OS ranged from 7 to 13 mo. For WHO-II and WHO-III meningiomas, the median OS ranged from 6 to 33 mo. The highest OS was achieved with erlotinib and gefitinib (2). A phase 2 trial of antiangiogenic sunitinib for recurrent and progressive WHO-II $(n=30)$ and WHO-III $(n=6)$ meningiomas reported a median OS of 24 mo (31). Similarly, a phase 2 trial of bevacizumab and everolimus for mixed WHO-I, WHO-II, and WHO-III meningioma patients reported a median OS of 23 mo (32). The present study found OS12 rates of $88 \%, 71 \%$, and $52 \%$ for WHO-I, WHO-II, and WHO-III meningiomas, respectively. The median OS was 43 mo, again indicating that SSTR-targeted PRRT could benefit patients with treatment-refractory meningiomas.

\section{Implications}

The findings of the present study have implications for clinical practice, the drafting of guidelines, health insurance reimbursement, and further research. To clinicians, SSTR-targeted PRRT represents a promising approach for treatment-refractory meningioma when all other therapies have failed. Thus, future guidelines should mention this therapeutic option for patients with treatmentrefractory meningiomas. Our results warrant controlled studies to validate the adverse effects and benefits of SSTR-targeted PRRT for treatment-refractory meningioma prospectively. Our data on response and survival will help to determine expected effects and sample sizes. Finally, future studies should adhere to 1 common radiologic assessment protocol to minimize heterogeneity and improve external validity.

\section{CONCLUSION}

To our knowledge, the present IPD meta-analysis represents the most comprehensive analysis of the benefits of and adverse events associated with SSTR-targeted PRRT for treatment-refractory meningioma. The treatment is well tolerated, achieves disease control in most cases, and shows promising results regarding PFS and OS. This treatment should be considered when other therapies have failed. 


\section{DISCLOSURE}

No potential conflict of interest relevant to this article was reported.

KEY POINTS

QUESTION: SSTR-targeted PRRT in treatment-refractory meningioma is conceptually attractive, but previously published studies included small numbers of selected patients.

PERTINENT FINDINGS: To our knowledge, the present IPD metaanalysis represents the most comprehensive analysis of the benefits of and adverse events associated with SSTR-targeted PRRT for treatment-refractory meningioma. The results demonstrate that the treatment is well tolerated, achieves disease control in most cases, and shows promising results regarding PFS and OS.

IMPLICATIONS FOR PATIENT CARE: SSTR-targeted PRRT should be considered when other therapies have failed.

\section{REFERENCES}

1. Louis DN, Perry A, Reifenberger G, et al. The 2016 World Health Organization Classification of Tumors of the Central Nervous System: a summary. Acta Neuropathol (Berl). 2016;131:803-820.

2. Kaley T, Barani I, Chamberlain M, et al. Historical benchmarks for medical therapy trials in surgery- and radiation-refractory meningioma: a RANO review. Neuro Oncol. 2014;16:829-840.

3. Goldbrunner R, Minniti G, Preusser M, et al. EANO guidelines for the diagnosis and treatment of meningiomas. Lancet Oncol. 2016;17:e383-e391.

4. Mirian C, Duun-Henriksen AK, Juratli T, et al. Poor prognosis associated with TERT gene alterations in meningioma is independent of the WHO classification: an individual patient data meta-analysis. J Neurol Neurosurg Psychiatry. 2020: 91:378-387.

5. Seystahl K, Stoecklein V, Schüller U, et al. Somatostatin receptor-targeted radionuclide therapy for progressive meningioma: benefit linked to ${ }^{68} \mathrm{Ga}$-DOTATATE/-TOC uptake. Neuro Oncol. 2016;18:1538-1547.

6. Gerster-Gilliéron K, Forrer F, Maecke H, Mueller-Brand J, Merlo A, Cordier D. ${ }^{90}$ Y-DOTATOC as a therapeutic option for complex recurrent or progressive meningiomas. J Nucl Med. 2015;56:1748-1751.

7. Stewart LA, Clarke M, Rovers M, et al. Preferred Reporting Items for Systematic Reviews and Meta-Analyses of individual participant data: the PRISMA-IPD Statement. JAMA. 2015;313:1657-1665.

8. Marincek N, Radojewski P, Dumont RA, et al. Somatostatin receptor-targeted radiopeptide therapy with ${ }^{90} \mathrm{Y}$-DOTATOC and ${ }^{177} \mathrm{Lu}$-DOTATOC in progressive meningioma: long-term results of a phase II clinical trial. J Nucl Med. 2015;56: 171-176.

9. Sterne JA, Hernan MA, Reeves BC, et al. ROBINS-I: a tool for assessing risk of bias in non-randomised studies of interventions. BMJ. 2016;355:i4919.

10. National Cancer Institute. Common Terminology Criteria for Adverse Events v3.0 (CTCAE). National Cancer Institute website. https://ctep.cancer.gov/protocol Development/electronic_applications/ctc.htm. Accessed on March 3, 2021.

11. National Cancer Institute. Common Terminology Criteria for Adverse Events v4.0 (CTCAE). National Cancer Institute website. https://ctep.cancer.gov/protocol Development/electronic_applications/ctc.htm. Accessed on March 3, 2021.

12. World Health Organization. WHO Handbook for Reporting Results of Cancer Treatment. Geneva, Switzerland: World Health Organization; 1979.

13. Higgins JPT, Thompson SG, Deeks JJ, Altman DG. Measuring inconsistency in meta-analyses. BMJ. 2003;327:557-560.
14. Ilhan H, Seystahl K, Stoecklein V, et al. Peptide receptor radionuclide therapy with Y-90 DOTATOC and Lu-177 DOTATATE in patients with progressive meningioma and prognostic value of pretherapeutic Ga-68 DOTATOC/-TATE PET/CT. J Nucl Med. 2016;57(suppl 2):631.

15. Kreissl MC, Hänscheid $\mathrm{H}$, Löhr $\mathrm{M}$, et al. Combination of peptide receptor radionuclide therapy with fractionated external beam radiotherapy for treatment of advanced symptomatic meningioma. Radiat Oncol. 2012;7:99.

16. Hänscheid H, Sweeney RA, Flentje M, et al. PET SUV correlates with radionuclide uptake in peptide receptor therapy in meningioma. Eur J Nucl Med Mol Imaging. 2012;39:1284-1288.

17. Minutoli F, Amato E, Sindoni A, et al. Peptide receptor radionuclide therapy in patients with inoperable meningiomas: our experience and review of the literature. Cancer Biother Radiopharm. 2014;29:193-199.

18. Bartolomei M, Bodei L, De Cicco C, et al. Peptide receptor radionuclide therapy with ${ }^{90}$ Y-DOTATOC in recurrent meningioma. Eur J Nucl Med Mol Imaging. 2009;36:1407-1416.

19. van Essen M, Krenning EP, Kooij PP, et al. Effects of therapy with $\left[{ }^{177} \mathrm{Lu}-\right.$ DOTA0, Tyr3] octreotate in patients with paraganglioma, meningioma, small cell lung carcinoma, and melanoma. J Nucl Med. 2006;47:1599-1606.

20. Kwekkeboom DJ, de Herder WW, Kam BL, et al. Treatment with the radiolabeled somatostatin analog [177 Lu-DOTA 0,Tyr3]octreotate: toxicity, efficacy, and survival. J Clin Oncol. 2008;26:2124-2130.

21. Umscheid CA, Margolis DJ, Grossman CE. Key concepts of clinical trials: a narrative review. Postgrad Med. 2011;123:194-204.

22. Jardim DL, Hess KR, Lorusso P, Kurzrock R, Hong DS. Predictive value of phase I trials for safety in later trials and final approved dose: analysis of 61 approved cancer drugs. Clin Cancer Res. 2014;20:281-288.

23. Julka PK, Doval DC, Gupta S, Rath GK. Response assessment in solid tumours: a comparison of WHO, SWOG and RECIST guidelines. $\mathrm{Br} J$ Radiol. 2008; 81:444-449.

24. Wen PY, Macdonald DR, Reardon DA, et al. Updated response assessment criteria for high-grade gliomas: response assessment in Neuro-Oncology Working Group. J Clin Oncol. 2010;28:1963-1972.

25. Wen PY, Cloughesy TF, Ellingson BM, et al. Report of the Jumpstarting Brain Tumor Drug Development Coalition and FDA clinical trials neuroimaging endpoint workshop (January 30, 2014, Bethesda MD). Neuro Oncol. 2014;16(suppl 7): vii36-vii47.

26. Mirian C, Skyrman S, Bartek J Jr, et al. The Ki-67 proliferation index as a marker of time to recurrence in intracranial meningioma. Neurosurgery. July 2, 2020 [Epub ahead of print].

27. Gousias K, Schramm J, Simon M. The Simpson grading revisited: aggressive surgery and its place in modern meningioma management. J Neurosurg. 2016; 125:551-560.

28. Imhof A, Brunner $\mathrm{P}$, Marincek $\mathrm{N}$, et al. Response, survival, and long-term toxicity after therapy with the radiolabeled somatostatin analogue $\left[{ }^{90} \mathrm{Y}\right.$ DOTA]-TOC in metastasized neuroendocrine cancers. J Clin Oncol. 2011;29: 2416-2423.

29. Bodei L, Kidd M, Paganelli G, et al. Long-term tolerability of PRRT in 807 patients with neuroendocrine tumours: the value and limitations of clinical factors. Eur J Nucl Med Mol Imaging. 2015;42:5-19.

30. Sabet A, Ezziddin K, Pape U-F, et al. Long-term hematotoxicity after peptide receptor radionuclide therapy with ${ }^{177}$ Lu-octreotate. J Nucl Med. 2013;54:18571861.

31. Kaley TJ, Wen P, Schiff D, et al. Phase II trial of sunitinib for recurrent and progressive atypical and anaplastic meningioma. Neuro Oncol. 2015;17:116-121.

32. Shih KC, Chowdhary S, Rosenblatt P, et al. A phase II trial of bevacizumab and everolimus as treatment for patients with refractory, progressive intracranial meningioma. J Neurooncol. 2016;129:281-288. 